\title{
Optimizing tube hydroforming using process simulation and experimental verification
}

\author{
Yingyot Aue-U-Lan, Gracious Ngaile, Taylan Altan* \\ ERC for Net Shape Manufacturing, The Ohio State University, 339 Baker Systems, 1971 Neil Ave, Columbus, OH 43210, USA
}

\begin{abstract}
The success of a tube hydroforming (THF) process is highly dependent on the loading paths (axial feed versus pressure) used. Finite element (FE)-based simulation was used to determine optimum loading paths for hydroforming of structural parts with different tubular materials. Experimental and simulation results have demonstrated that FE-based loading paths can significantly reduce trial and error, enhance productivity and expand the THF capability in forming complex parts. The test results also demonstrated that the reliability of the FE-based loading paths is highly dependent on the accuracy of the material properties of the blank, interface friction, and how close the properties of the welding zone are to the base material of the tubular blank.
\end{abstract}

(C) 2003 Elsevier B.V. All rights reserved.

Keywords: Tube hydroforming; FEM; Simulation

\section{Introduction}

Tube hydroforming (THF) is now widely used in making tubular parts of different configurations used in automotive industry, household appliances, and other applications. The rapid growth of this technology has been due to the advantages THF offers compared to conventional manufacturing via stamping and welding, namely (a) part consolidation; (b) weight reduction through more efficient section design and tailoring of the wall thickness in structural components; (c) improved structural strength and stiffness via optimized section geometry; (d) lower tooling costs due to fewer parts; (e) fewer secondary operations (less welding and punching of holes during hydroforming); (f) tighter tolerances and reduced springback that facilitates assembly and $(\mathrm{g})$ reduced scrap since trimming of excess material is far less in THF than in stamping [1].

The success of a THF process is, however, dependent on a number of variables such as the loading paths (internal pressure versus time and axial material feed versus time), lubrication conditions, and material formability. A suitable combination of all these variables is of paramount importance to avoid part failure due to wrinkling, pinching, buckling, or bursting. Depending on the complexity of the part, the THF process window can be very small thus making it difficult to obtain the right loading paths. Hence, it

\footnotetext{
* Corresponding author.

E-mail address: altan.1@osu.edu (T. Altan).
}

is imperative to establish a systematic way for determining loading paths the finite element analysis (FEA).

Traditionally, the loading paths are determined by an iterative "trail-and-error" process and rely on past experience and simple equation. For example, axial feed for a specific part can be estimated by using volume constancy and assuming that the wall thickness of the formed part remains constant. In reality, however, it is almost impossible to maintain constant wall thickness due to the presence of frictional stress at the tool-tube interface and the variations in material flow over the entire deformation zone. The pressure loading path is estimated by relating three pressure components, namely, the yield pressure of the material $P_{\text {yield }}$; the bursting pressure $P_{\text {bursting }}$ and the calibration pressure

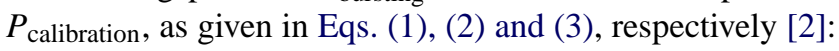

$$
\begin{aligned}
& P_{\text {yield }}=\sigma_{\mathrm{y}} \frac{2 t_{0}}{D_{0}-t_{0}} \\
& P_{\text {bursting }}=\sigma_{\mathrm{u}} \frac{4 t_{0}}{D_{\mathrm{p}}-t_{0}} \\
& P_{\text {calibration }}=\frac{2}{\sqrt{3}} \sigma_{\mathrm{f}}\left[\ln \frac{r_{\mathrm{b}}}{r_{\mathrm{b}}-t}\right]
\end{aligned}
$$

where $t_{0}$ is the initial tube wall thickness, $D_{0}$ the tube diameter, $D_{\mathrm{p}}$ the protrusion diameter, $r_{\mathrm{b}}$ the smallest die corner radius, $\sigma_{\mathrm{f}}$ the flow stress of the material, $\sigma_{\mathrm{y}}$ the yield strength of the material and $\sigma_{\mathrm{u}}$ the ultimate tensile strength of the material.

From these three pressure components, a linear pressure curve can be constructed. However, for complex parts the 
estimated linear pressure curve may not agree with the process window, thus requiring expensive and time consuming trial-and-error based iterative procedures.

Considerable research has been conducted to find robust and cost effective techniques to determine optimal loading paths. Three FE-based techniques have been proposed in the literature: self-feeding (SF) [3]; optimization [4] and adaptive simulation (AS) [5]. In the present study, experimental verifications of two approaches (SF and AS techniques) are discussed.

\section{Loading path determination via $\mathrm{SF}$ and adaptive FE simulations}

\subsection{SF approach}

This is a method designed to restrict the search for the loading path to a proper family of curves and select the optimum within that family. This method contains two steps. The first step is used to determine the relationship between internal pressure $(P)$ and axial feed $\left(d_{\mathrm{ax}}\right)$, where the process is simulated by imposing only the internal pressure versus time. The friction at the interface is assumed to be zero. Then, the displacement versus time at the node located at the ends of the tube and the maximum thinning on the deforming tube are determined. This information is used to estimate approximately how much the axial feed should be in order to avoid excessive thinning of the hydroformed tube. In the second simulation step, a friction coefficient is prescribed and the axial feed is increased by a certain amount using a scale factor, $\alpha\left(\alpha^{*} \mathrm{SF}\right)$, as shown in Fig. 1. This scale factor is varied until a successful part is formed [6,7].

\subsection{AS approach}

The principle idea of the AS method is to feed the material into the deformation zone as much as possible without any wrinkles or fracture. At the beginning of the simulation, the tube is "deformed" by pressuring to the yield pressure $\left(P_{\text {iy }}\right)$. Then, axial feeding is provided in the simulation, while maintaining the pressure at $P_{\text {iy }}$, until wrinkles are detected. The wrinkles are then eliminated by pressuring the tube without any axial feeding. Once the wrinkles are eliminated,

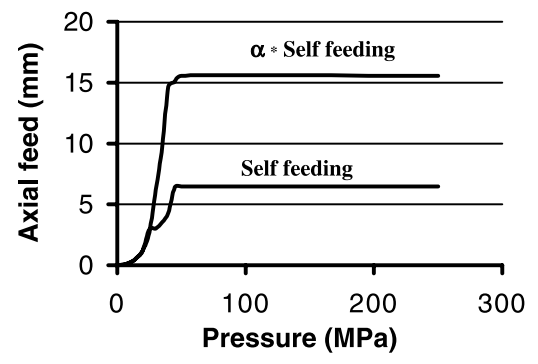

Fig. 1. SF loading paths: $\alpha$ is a scale factor to increase the amount of axial feeding.
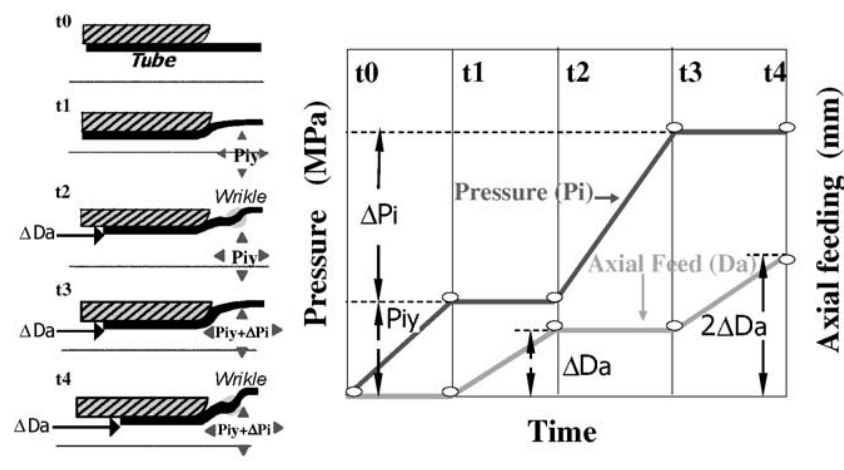

Time

Fig. 2. Schematic procedure of the AS $\left(P_{\mathrm{i}}\right.$ : internal pressure, $\Delta P_{\mathrm{i}}$ : internal pressure increment, $P_{\text {iy }}$ : yield pressure, $\Delta D_{\mathrm{a}}$ : axial feed increment).

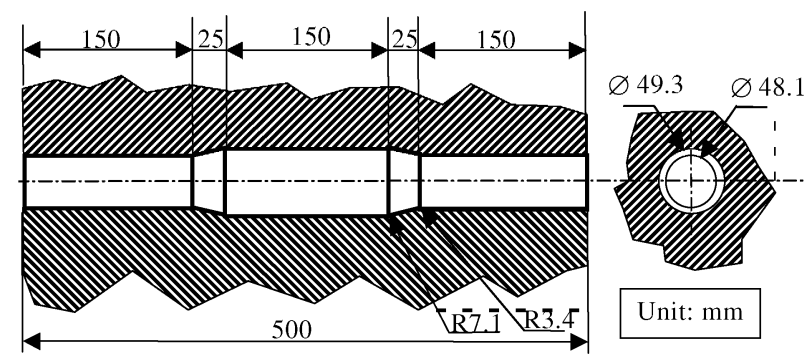

Fig. 3. THF structural part.

the tube is fed by axial feeding at a constant pressure (see Fig. 2). These steps are repeated until a part without wrinkles and excessive thinning is obtained.

These two methods (SF and AS) were used to determine loading paths for the hydroforming of an axisymmetric part shown in Fig. 3. Three different tube materials were used in this study. The flow stress $(\bar{\sigma})$ of these materials was expressed in form of $\bar{\sigma}=K\left(\varepsilon_{0}+\bar{\varepsilon}\right)^{n}$. The coefficients of this expression are given in Table 1. Commercial finite element software DEFORM and PAMSTAMP were used for the determination of the loading paths. In the simulations, a friction coefficient of 0.05 was used. Figs. 4-6 show some of the loading paths for stainless steel tubing AISI 304, low carbon steel tubing STK 400 Japanese standard (JIS) and STM 12A (JIS), respectively.

\subsection{Fracture criterion}

In the process of determining the loading paths using the methods discussed above, a fracture criterion had to be included in the FEA in order to identify "when" and "where"

Table 1

Dimensions and mechanical properties obtained by hydraulic bulge test for the tubular materials used in this study

\begin{tabular}{llllll}
\hline Material & OD $(\mathrm{mm})$ & $t_{0}(\mathrm{~mm})$ & $K(\mathrm{MPa})$ & $n$ & $\varepsilon_{0}$ \\
\hline AISI 304 & 48.6 & 2.2 & 1555 & 0.615 & 0.03 \\
STK 400 & 48.6 & 2.3 & 581 & 0.142 & 0.01 \\
STM 12A & 48.6 & 2.9 & 720 & 0.161 & 0.01 \\
\hline
\end{tabular}




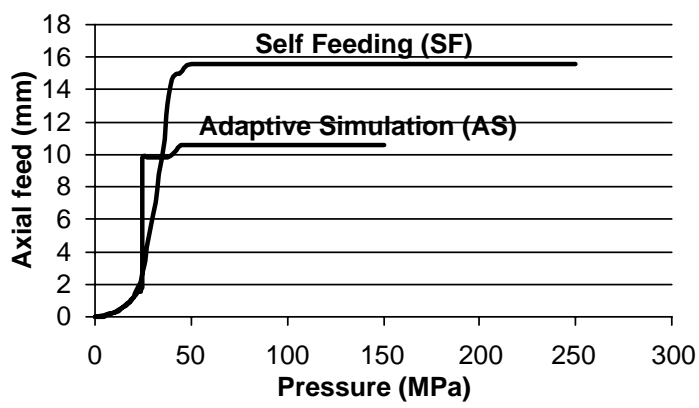

Fig. 4. Axial feed versus pressure curves (SF and AS loading paths for AISI 304 tubing).

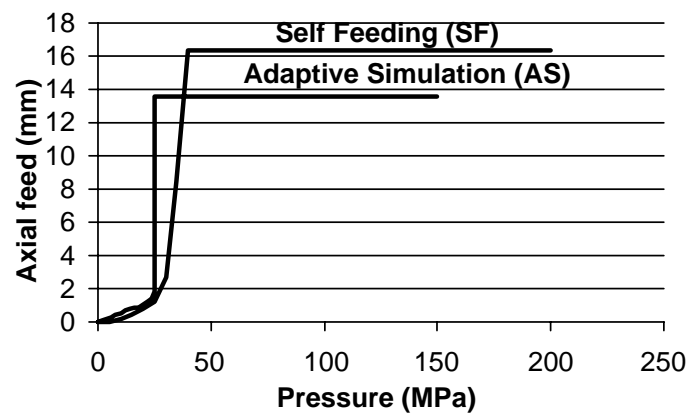

Fig. 5. Axial feed versus pressure curves (SF and AS loading paths for STK 400 tubing).

the fracture will occur. In this study, maximum thinning, $t_{\mathrm{f}}$, was used as a fracture criterion. The maximum thinning was calculated by using the thickness strain $\left(\varepsilon_{t}\right)$ at the planestrain condition of the forming limit diagram (FLD), Eq. (4):

$\varepsilon_{t}=\ln \left(\frac{t_{\mathrm{f}}}{t_{0}}\right) \quad$ or $\quad t_{\mathrm{f}}=t_{0} \exp \left(\varepsilon_{t}\right)$

where $t_{0}$ is the initial tube wall thickness and $t_{\mathrm{f}}$ the final tube wall thickness.

The major engineering strain, $e_{\theta}$, at the plane-strain condition was approximated by using the North American Deep Drawing Research Group (NADDRG) empirical equation [8], as shown in Eq. (5):

$e_{\theta}=\mathrm{FLD}_{0}=\left(23.3+\frac{360}{25.4} t_{0}\right)\left(\frac{n}{0.21}\right)$

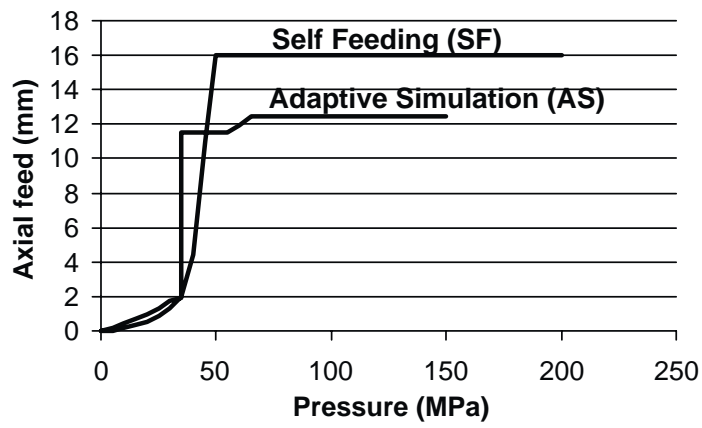

Fig. 6. Axial feed versus pressure curves (SF and AS loading paths for STM 12A tubing). where $\mathrm{FLD}_{0}$ the engineering major strain at plane-strain condition, $n$ the strain-hardening exponent, $e_{\theta}$ the engineering strain along the hoop direction (major strain), $t_{0}$ the initial tube wall thickness, or sheet thickness. The engineering major strain in plane-strain condition was converted to true major strain by using Eq. (6):

$\varepsilon_{\theta}=\ln \left(1+e_{\theta}\right)$

By assuming volume constancy Eq. (7) and $\varepsilon_{Z}=0$ (plane-strain condition), the true strain in the thickness direction was determined, Eq. (8):

$\varepsilon_{\theta}+\varepsilon_{Z}+\varepsilon_{t}=0$

$\varepsilon_{t}=-\varepsilon_{\theta}$

$\%$ thinning $=\frac{t_{0}-t_{\mathrm{f}}}{t_{0}}$

where $\varepsilon_{Z}$ is the true strain along the longitudinal direction (minor strain), $\varepsilon_{t}$ the true strain in the thickness direction (thickness strain) and \% thinning is the maximum tube wall thinning in percentage. By relating (4)-(8) the failure limit or the minimum tube wall thickness prior to fracture, $t_{\mathrm{f}}$, can be determined.

\section{THF experiments of a structural part}

All experiments were conducted at Kawasaki Hydromechanics Corporation, Akashi-Hyogo, Japan. The press used for the tests has a capacity of $10,000 \mathrm{kN}$ with an axial cylinder pressure of up to $300 \mathrm{MPa}$ ( 3000 bars) and a punch force of up to $1000 \mathrm{kN}$. The in-built process controls in the press include: (a) input variables: pressure and axial feed versus time (independent feed controls in each cylinder) and (b) output variables: actual pressure, actual feed, cylinder force, instantaneous fluid volume inside the deforming tube.

\subsection{Experimental procedures}

The tubes were cut to $440 \mathrm{~mm}$ length and the ends of the tubes were trimmed and deburred to secure the required size (Fig. 3). All the specimens were lubricated using molybdenum disulfide. The dies were cleaned (fluid medium removed) before putting the specimens into the die cavity. After placing the specimens into the die cavity, the loading paths (axial feed versus time, and pressure versus time) were entered into the control panel of the press ready for the test. After the tests, the specimens were inspected for surface quality and defects such as wrinkling, buckling and bursting. Finally, tube wall thickness distribution measurements were made using an ultrasonic device.

\subsection{FE simulation and experimental results for AISI 304 tubing}

As shown in Figs. 7 and 8, both SF and AS loading paths produced good hydroformed parts when AISI 304 tubing 


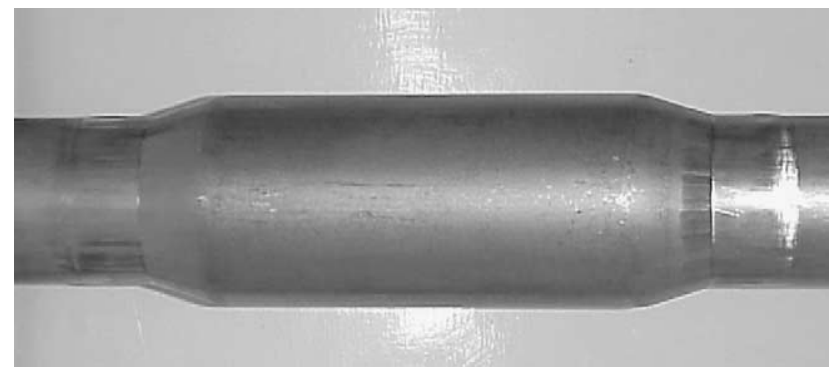

Fig. 7. Hydroformed part from AISI 304 using the loading paths obtained by SF.

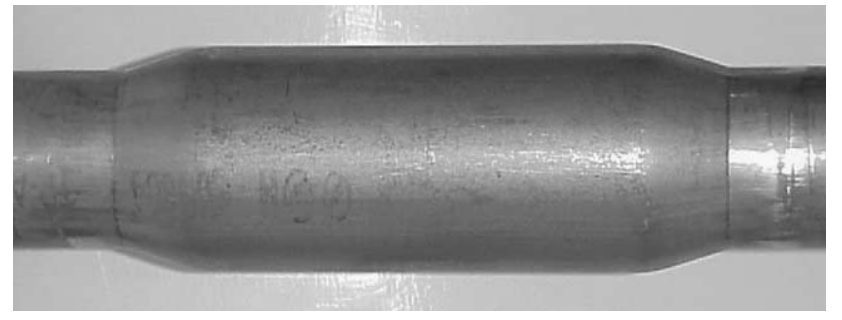

Fig. 8. Hydroformed part from AISI 304 using the loading paths obtained by AS.

was used. It should be noted, however, that three loading paths were tried for SF. The loading path shown in Fig. 4 was successful, but the other two loading paths resulted in leakage of the pressure medium.

Fig. 9 shows the tube wall thinning distribution given in percentage for both hydroformed parts from SF and AS methods. It is interesting to find that a higher percentage tube wall thinning is observed for AS. The fact that SF produced a good part and with lower percentage tube wall thinning than AS implies that there is a possibility of reducing the percentage tube wall thinning even further. In other words, the loading path determined by AS was not optimal. This may be attributed to the inaccuracy of material properties and the friction coefficient $(\mu=0.05)$ assumed in the simulations.

Figs. 10 and 11 show comparisons of wall thickness distribution in the longitudinal direction between experiments and FE simulations for the SF and AS methods,

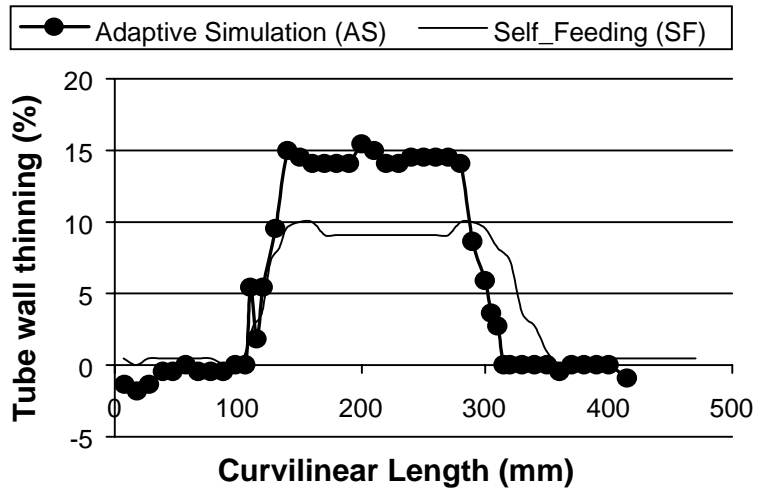

Fig. 9. Calculated wall thinning distributions along the longitudinal direction of the part for AISI 304.

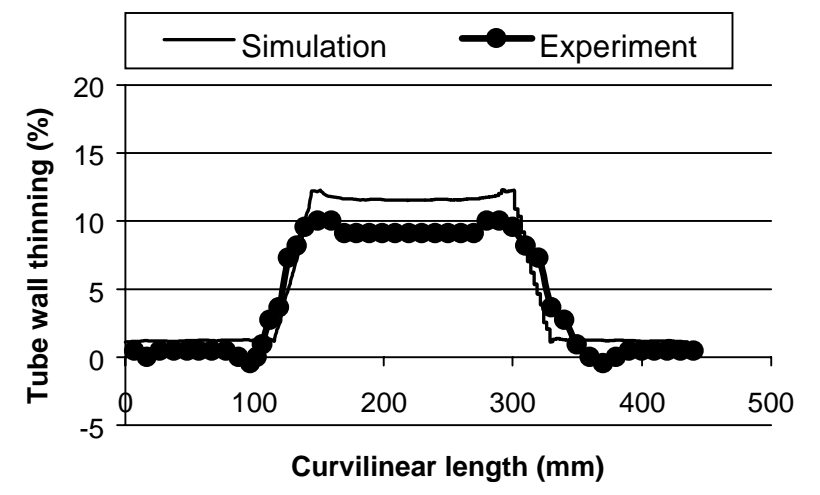

Fig. 10. Comparison of thinning distribution along the longitudinal direction between the simulation and experiment for AISI 304 tubing (SF method).

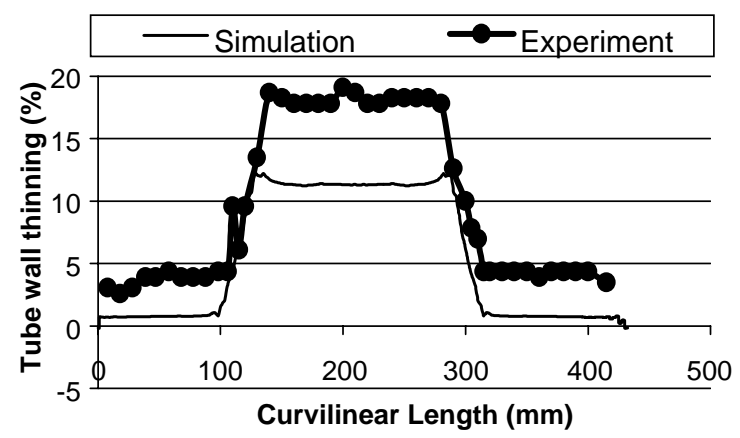

Fig. 11. Comparison of thinning distribution along the longitudinal direction between the simulation and experiment for AISI 304 tubing (AS method).

respectively. The simulations were found to be in good agreement with experiments. The difference in the maximum percentage thinning between experiment and simulation is about 2.3 and $33 \%$ for SF and AS, respectively.

\subsection{FE simulation and experimental results for STK 400 (JIS)}

The experiments on STK 400 tubing were conducted using the loading paths given in Fig. 5. It is seen that only SF produced a good part (Fig. 12). While only $10 \%$ thinning is observed for SF, the wall thinning percentage for AS went as high as $20 \%$, producing wrinkles and fracture (Figs. 13 and 14).

Several experiments were also conducted such that the test was stopped at a particular pressure in order to verify

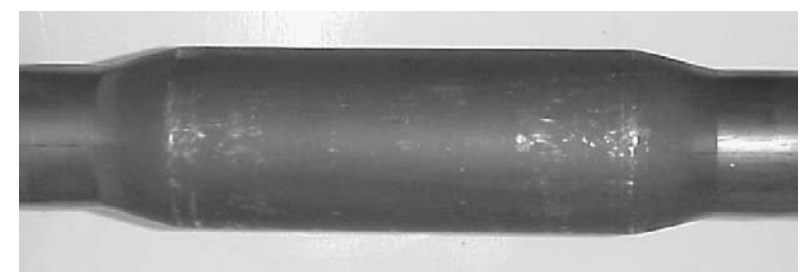

Fig. 12. Hydroformed part for STK 400 (SF method). 


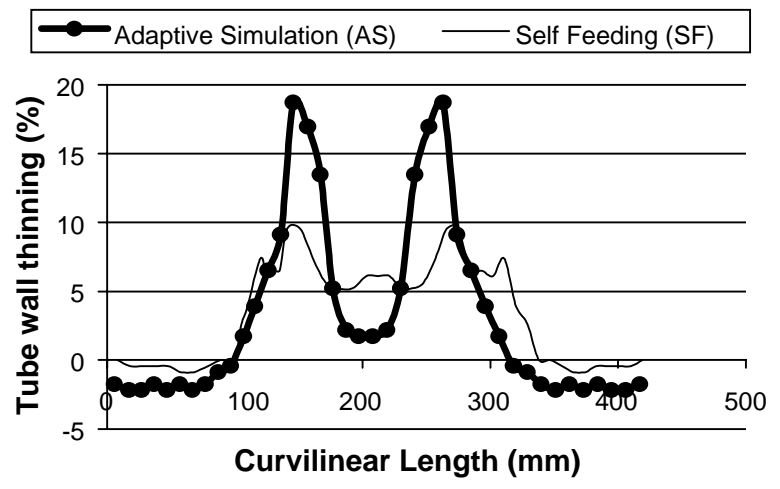

Fig. 13. Simulated thinning distributions along the longitudinal direction of the part for STK 400.

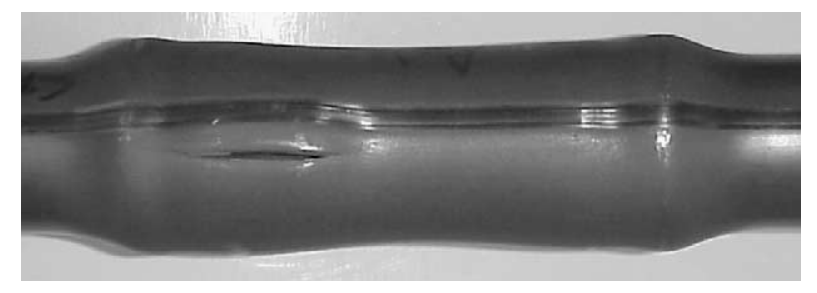

Fig. 14. Hydroformed part for STK 400 (AS method).

the accuracy of the FE simulation to predict a wrinkle. For example, Fig. 15 shows the deformed tube with wrinkles when the internal pressure reached $40 \mathrm{MPa}$. FE simulations also show that wrinkles occurred at this pressure although the amplitude of the wrinkles in the simulation was relatively smaller than that observed in the experiment.

Fig. 16 shows a comparison of thinning distribution in the longitudinal direction between the FE simulation and experiment conducted by using loading paths obtained by SF. About $6 \%$ difference was observed, which may be attributed to the inaccuracy of the flow stress, the effect of the weld line on deformation behavior, etc. as discussed later in Section 4.
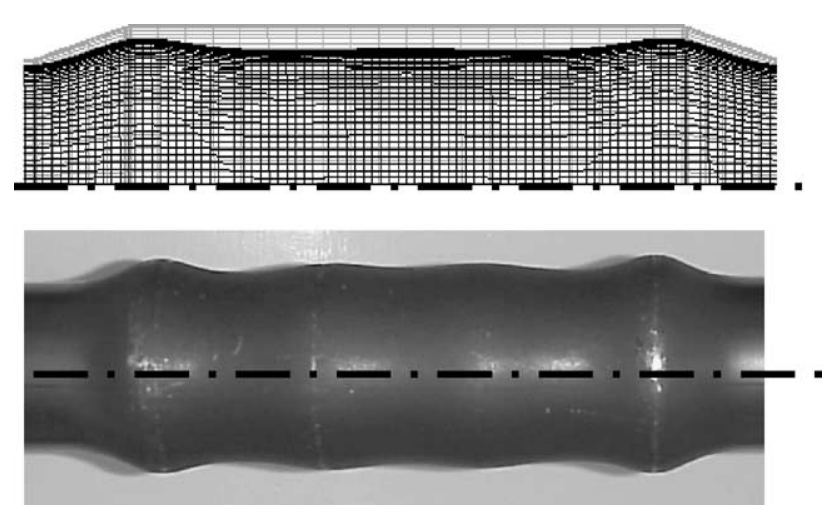

Fig. 15. Wrinkles occurring in the simulation (top) and experiment (bottom) for STK 400 tubing at $P=40 \mathrm{MPa}$.

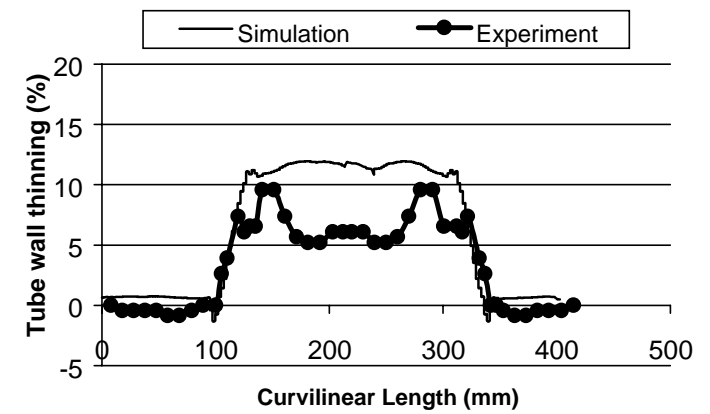

Fig. 16. Comparison of thinning distribution along the longitudinal direction between the simulation and experiment for STK 400 tubing (SF method).

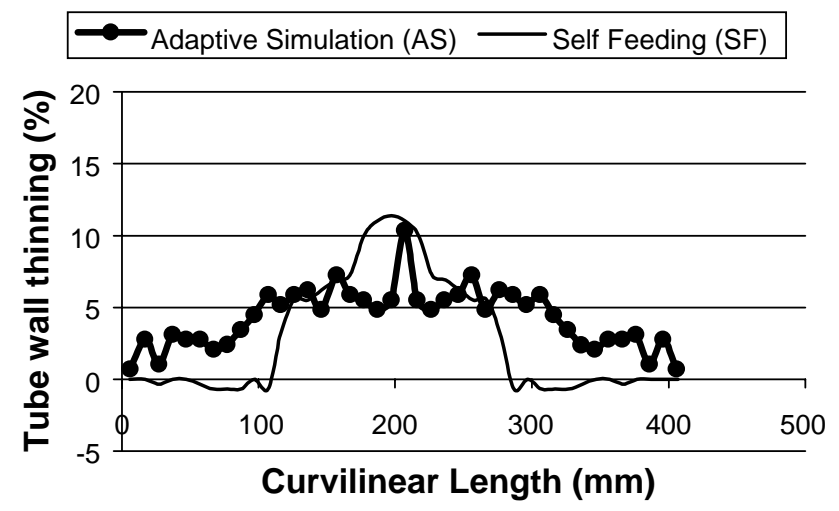

Fig. 17. Simulated thinning distribution along the longitudinal direction of the part for STM 12A.

\subsection{FE simulation and experimental results for STM $12 A(J I S)$}

SF and AS loading paths shown in Fig. 6 were applied to hydroform STM 12A tubing (Fig. 17). However, none of the loading paths could successfully form this material. Figs. 18 and 19 show the fractured and wrinkled parts, respectively. It should be noted that the wall thickness for this tube was $2.9 \mathrm{~mm}$ and the outside diameter was $48.6 \mathrm{~mm}$, resulting in

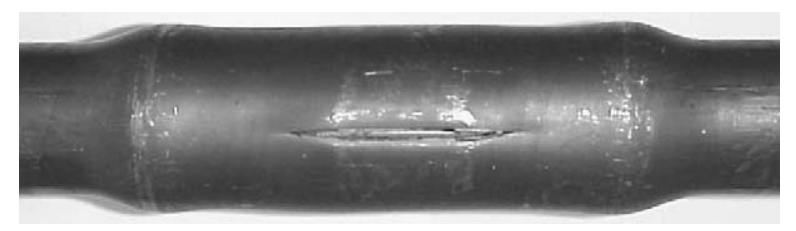

Fig. 18. Fractured hydroformed part for STM 12A (SF method).

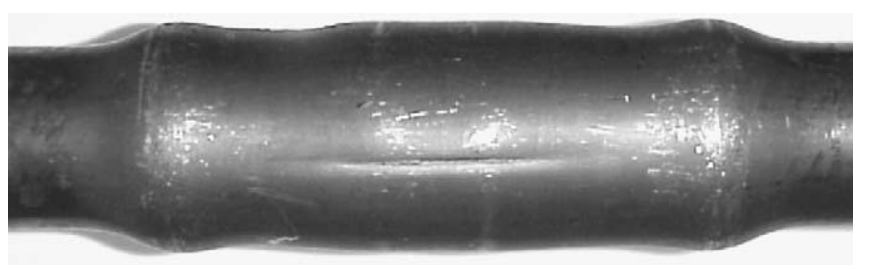

Fig. 19. Wrinkled hydroformed part for STM 12A (AS method). 


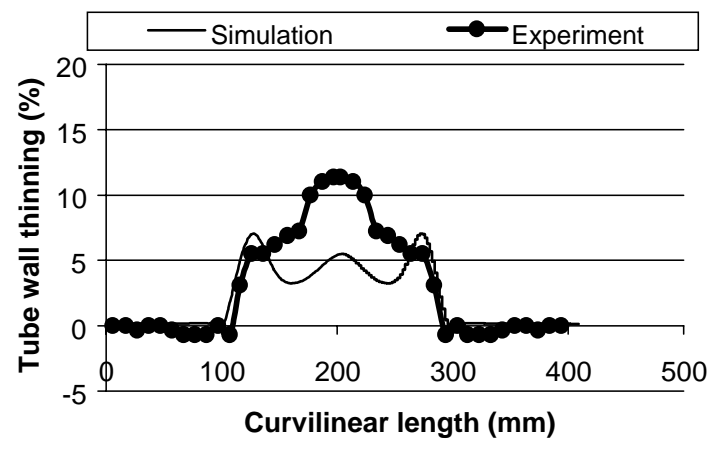

Fig. 20. Comparison of the thinning distributions along the longitudinal direction between the simulation and experiment for STM 12A tubing.

a diameter to thickness ratio of around 17. This value is rather low and can result in substantial error in wall thickness predictions using shell elements in the FE simulation code (PAMSTAMP uses 4-noded shell elements). The shell elements can deliver accurate results for geometries with a diameter to thickness ratio of above 20 because the bending effects can be neglected for these ratios. Since the current tube geometry is such that the diameter to thickness ratio falls below 20, the thickness predictions (Fig. 20) deviate from the experimental observations.

\section{Discussion}

The experimental results obtained in this study have demonstrated that the determination of loading paths for the THF by SF and AS methods can enhance productivity, reduce scrap rate and significantly reduce the number of trials used for producing a sound part by THF. However, it is important that accurate material data (flow stress), and friction conditions are used as input to the FE simulations.

The tests on stainless steel tubing AISI 304 presented above were successful for both SF and AS. It is of interest to note that when another batch of AISI 304 obtained from a different vendor was subjected to the same test conditions, no successful parts could be formed. This indicates that it is necessary to know the deformation history of the tubes used in THF.

The common practice of using the flow stress data obtained by a tensile test of a sheet metal specimen for THF process simulations is not correct because (a) the state of stress encountered in the hydroforming process (biaxial) is different from that encountered in the tensile test (uniaxial tension) and (b) the operations for making a tube from a sheet specimen (e.g., roll forming) induce some strain in the tube thereby strain-hardening it along the circumference. For these two reasons, the flow stress of the tube specimen is most likely to be different from that of the sheet specimen from which the tube was manufactured. It is therefore appropriate to determine the flow stress of a tube by using the bulge test [9]. Even with such a test, factors pertaining to tube manufacturing need to be given special attention.

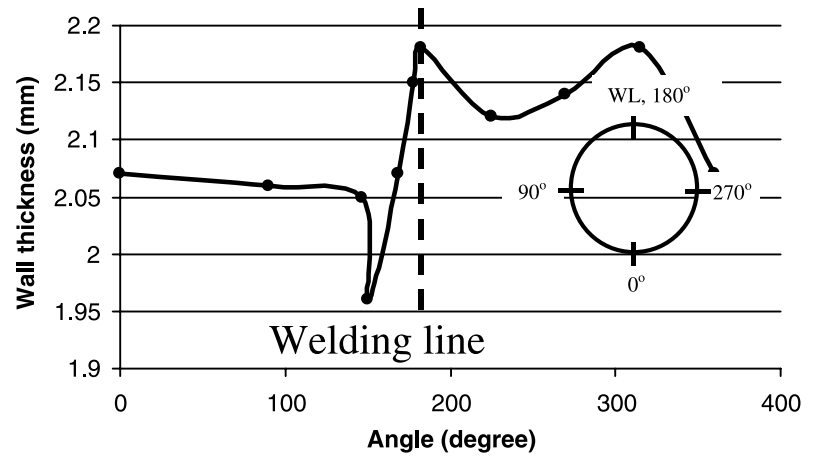

Fig. 21. Thickness distribution around the circumferential direction of STK 400 tubing after THF. Burst location is at $170^{\circ}$ (AS loading path).

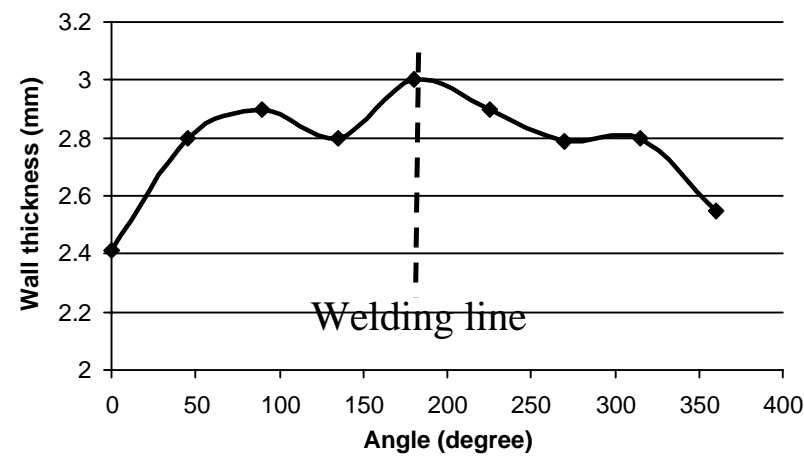

Fig. 22. Thickness distribution around the circumferential direction of STM 12A tubing after THF. Burst location is $180^{\circ}$ from the welding line (SF loading path).

In this study it was found that STM 400 and STK12A tubing could not be hydroformed using the AS approach. Upon analysis of the failed parts, it was found that the wall thickness distribution along the circumference of the tube was non-uniform despite the axisymmetrical nature of the part formed (Figs. 21-23).

It is assumed that one of the reasons for uneven and non-symmetrical thickness distribution is the effect of the welding line on the averaged flow stress of the tube material used in FEA. A clear understanding of the characteristic of

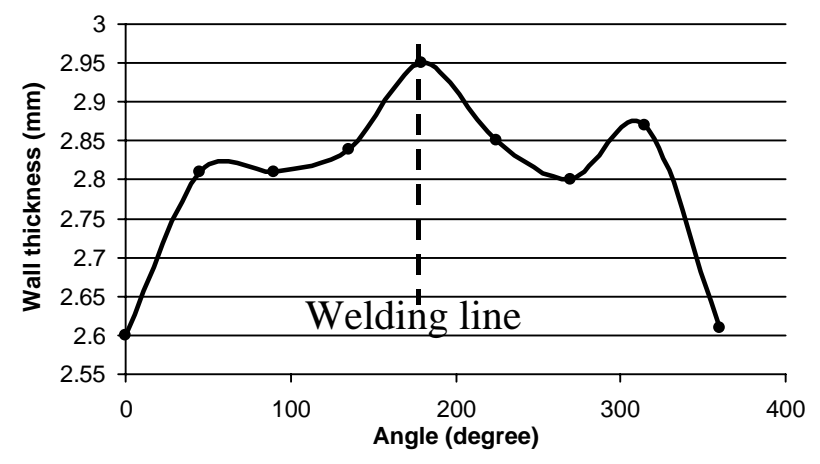

Fig. 23. Thickness distributions around the circumferential direction of STM 12A tubing after THF. Burst location is $180^{\circ}$ from the welding line (AS loading path). 


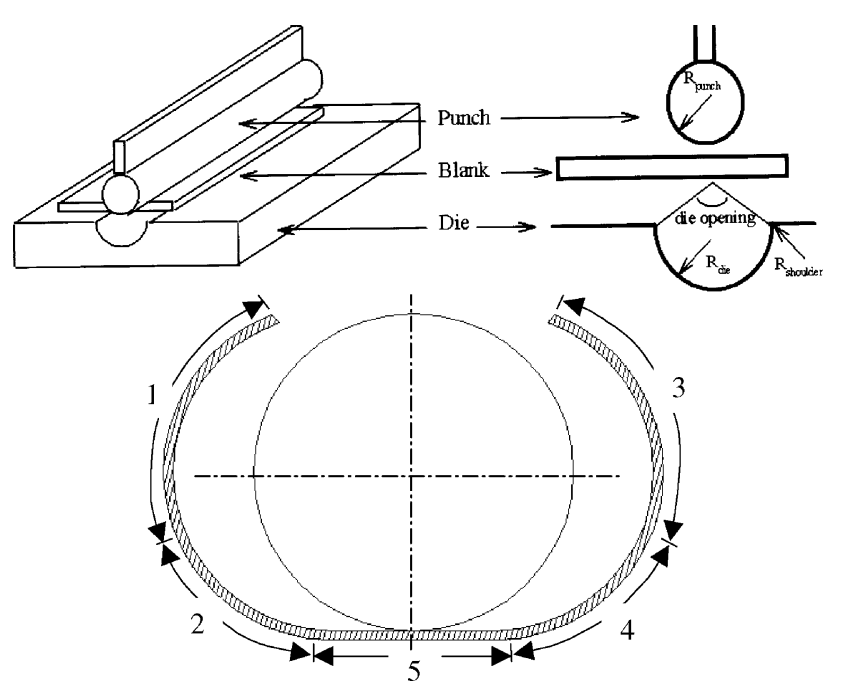

Fig. 24. Tube making by press forming.

the weld and the heat-affected zone is essential. Experimental studies from the literature show that the work hardening index of the weld and heat-affected zone is lower than the mean value for the base material, while the strength coefficient is higher [10]. A study is underway at ERC/NSM to address the effect of the welding line on the flow stress of the tube.

The roll forming operation, commonly used for tube manufacturing, imparts a non-uniform strain distribution on a tube, primarily in the circumferential direction.

An alternative to the rolling process is the press forming method shown in Fig. 24 [11]. With this method, the plastic strain generated during the bending process is evenly distributed over the entire tube with small variations in the radial direction.

\section{Summary and conclusions}

THF experiments were carried out to gather information on the reliability of THF loading paths determined by FEA. The major conclusions drawn from this study are:

1. The SF is a "systematic trial-and-error" approach for establishing a family of loading paths via FEA. The THF experiments done using this approach have shown that SF can significantly reduce the number of trial runs necessary for process development.

2. Both the SF and AS techniques have shown that the accuracy of the tube material data is of paramount importance for determining reliable loading paths.
3. The methods used in tube making and the properties of the material at or near the weld zone can have a tremendous effect on the flow of material in the die cavity due to uneven pre-strain distribution or change in the strain-hardening index and strength coefficient of the weld zone compared to those of the base material.

\section{Acknowledgements}

This study was partially supported by Tube Hydroforming Consortium conducted at ERC/NSM of the Ohio State University. Experiments were conducted at the Kawasaki Hydromechanics Corp in Japan. Mr. Khaled Tibari conducted some of the FE simulations. These contributions are gratefully acknowledged.

\section{References}

[1] F. Dohmann, C. Hartl, Hydroforming-a method to manufacture lightweight parts, J. Mater. Process. Technol. 60 (1996) 669676.

[2] T. Altan, S. Jirathearanat, M. Strano, Adaptive FEM process simulation for hydroforming tubes, in: Proceedings of the International Conference on Hydroforming, Fellbach-Stuttgart, Germany, November 2001.

[3] K. Tibari, Adaptive simulation in tube hydroforming, ERC/NSM Report No. THF/ERC/NSM-00-R-10, 2000.

[4] J.B. Yang, et al., Design sensitivity analysis and optimization of the hydroforming process, J. Mater. Process. Technol. 113 (2001) 666672.

[5] M. Strano, et al., Adaptive FEM simulation for tube hydroforming: a geometry-based approach for wrinkle detection, in: Proceedings of the 51st Annals of CIRP, 2001.

[6] M. Strano, et al., Virtual process development in tube hydroforming, J. Mater. Process. Technol., submitted for publication.

[7] M. Strano, S. Jirathearanat, Adaptive simulation concept for tube hydroforming, in: Proceedings of the Innovation in Tube Hydroforming Technology, Troy, June 2000.

[8] S.B. Levy, A comparison of empirical forming limit curves for low carbon steel with theoretical forming limit curves of Ramaekers and Bongaerts, IDDRG WG3, Ungarn, 1996.

[9] Y. Aue-u-lan, et al., Determination of flow stress and formability of tubes for hydroforming, ERC/NSM Report No. THF/ERC/NSM00-R-11, 2000.

[10] B.Y. Ghoo, Y.T. Keum, Y.S. Kim, Evaluation of the mechanical properties of welded metal in tailored steel sheet welded by $\mathrm{CO}_{2}$ laser, J. Mater. Process. Technol. 113 (2001) 692-698.

[11] B.D. Carleer, FE process simulation for tube hydroforming, Starting the tube forming process, in: Proceedings of the International Conference on Hydroforming, Fellbach-Stuttgart, Germany, November 2001. 